Int. J. Dev. Biol. 51: 247-253 (2007)

doi: $10.1387 / \mathrm{ijdb} .0622181 \mathrm{w}$

Developmental Expression Pattern

\title{
Spatiotemporal expression of the creatine metabolism related genes agat, gamt and ct1 during zebrafish embryogenesis
}

\author{
LIFENG WANG, YING ZHANG, MING SHAO and HONGWEI ZHANG* \\ Institute of Developmental Biology, Life Science College, Shandong University, Key Lab of Experimental Teratology of \\ the Ministry of Education, Jinan, China
}

\begin{abstract}
Glycine amidinotransferase (AGAT or GATM), guanidinoacetate methyltransferase (GAMT) and creatine transporter (CT1) are three proteins involved in the synthesis and uptake of creatine. The expression patterns of these three genes were examined in zebrafish embryos by whole mount in situ hybridization followed by histological sectioning. Expression of agat first appeared in the yolk syncytial layer (YSL) at the gastrula stage and was progressively up regulated during gastrulation. As development proceeds, agat was expressed in the mature somites during the segmentation stage and in the liver at $48 \mathrm{hpf}$. gamt showed a similar expression pattern to that of agat during embryogenesis. It was first detected in the center of the yolk from the cleavage to the gastrula stage. At the bud stage, its expression shifted to the YSL. gamt was also transiently expressed in the mature somites from $16 \mathrm{hpf}$ to $24 \mathrm{hpf}$ and became strongly expressed in the liver and in epithelial cells of the gut at $\mathbf{4 8} \mathbf{~ h p f . ~ c t ~} 1$ was initially uniformly expressed from the cleavage to the early segmentation stage; it was then strongly expressed in all the somites till $30 \mathrm{hpf}$ and in the gut of $48 \mathrm{hpf}$ embryos. However, ct 1 transcripts also appeared in the central nervous system during the segmentation stage, but not in the YSL, the yolk or the liver. Our data reveal for the first time distinct and unique patterns of expression of the creatine metabolism genes agat, gamt and ct1 during zebrafish embryogenesis.
\end{abstract}

KEY WORDS: agat, gamt, ct1, zebrafish, developmental expression

The creatine metabolism plays a crucial role for keeping the normal life of vertebrates. Endogenous creatine is synthesized by a two-step mechanism involving two enzymes: glycine amidinotransferase (AGAT or GATM) and guanidinoacetate methyltransferase (GAMT). Creatine is taken up by cells through CT1, a specific creatine transporter (Wyss and KaddurahDaouk, 2000). Defects of AGAT, GAMT and CT1 result in three kinds of creatine deficiency syndromes (CDS) occurring mostly in children (Schulze, 2003). The common clinical feature of three CDS is developmental delay/regression, mental retardation and severe disturbance of their expressive and cognitive speech (van der Knaap et al., 2000). The biochemical characteristics of CDS include severe depletion of creatine/phosphocreatine in the brain, as well as changes in creatine and creatinine concentrations in body fluids (Verhoeven et al., 2000, Cecil et al., 2001, Schulze, 2003, Stromberger et al., 2003, Almeida et al., 2004, Sykut-Cegielska et al., 2004). GAMT deficiency is characterized by accumulation of guanidinoacetic acid in brain and body fluids and shows intractable seizures and the movement disorder (Sykut-Cegielska et al., 2004). GAMT and AGAT deficiency have autosomal-recessive traits, whereas the CT1 defect is an X-linked disorder (Mancini et al., 2005, Leuzzi et al., 2006). Treatment with oral creatine supplementation is in part successful in GAMT and AGAT deficiency (Battini et al., 2006, Schulze et al., 2006), whereas it does not work on CT1 deficiency. Recently, it has been demonstrated that AGAT, GAMT and CT1 are expressed in many embryonic tissues during rat embryogenesis (Braissant et al., 2005). Here we carried out whole mount in situhybridization followed by histological sectioning to determine the spatiotemporal expression patterns of agat, gamt and ct 1 during zebrafish embryogenesis.

\section{Sequences and phylogenetic analyses of zebrafish AGAT, GAMT and CT1}

Three cDNAs (GenBank accession numbers AAH56747,

Abbreviations used in this paper:AGAT (or GATM), glycine amidinotransferase; GAMT, guanidinoacetate methyltransferase; CDS, creatine deficiency syndromes; CT1, creatine transporter 1; YSL, yolk syncytial layer.

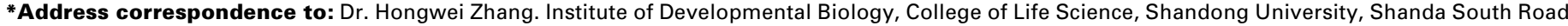
27th, Jinan, Shandong 250100, P.R. China. Fax: +86-531-8856-5610. e-mail: zhw@sdu.edu.cn
} 
A

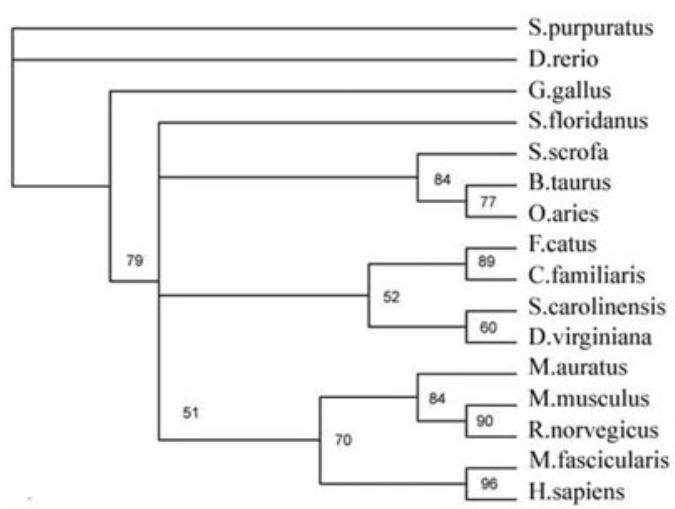

B

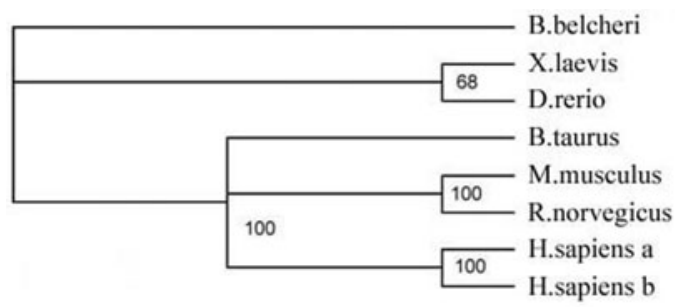

C

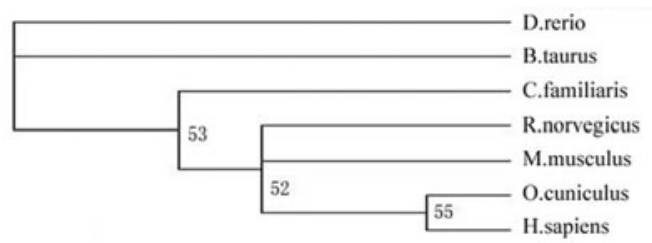

pancreas, CNS, skeletal muscles and intestine of rat embryos. In Xenopus, agat (xat) transcripts were detected around the yolk plug of early gastrula and strong expression was found in the notochord and the midline of the neural plate of early neurula. At tailbud stage, the expression was found both in the notochord and the trunk region (Zhao et al., 2001).

\section{Spatiotemporal expression of gamt}

Transcripts of gamtwere first detected in the central area of the yolk from cleavage (Fig. 3A) to gastrula stage (not shown). They were progressively expanded in the YSL at bud and early segmentation stages, while no transcript was detected in other
Fig. 1. Phylogenetic tree constructed using Treepuzzle 5.0 software according to the alignment of the protein sequences of AGAT(A), GAMT (B) and CT1 (C) in zebrafish and other species. The numbers after the internal branches indicated the bootstrap value (value/1000) for each group. Zebrafish AGAT and CT1 fell outside the other vertebrate groups and formed an independent clade in the trees respectively, while zebrafish GAMT and frog GAMT were in the same clade.

NM_205741 and XM_690840) encoding zebrafish AGAT, GAMT and CT1 were identified in the database. The deduced proteins were 422, 234 and 652 amino acids respectively, showing high levels of conservation to known AGAT, GAMT and CT1 proteins in human and other vertebrates, ranging from $65 \%$ to $86 \%$ overall identities (Appendix 1). Phylogenetic analyses were performed by the neighbor-joining analysis. Zebrafish AGAT and CT1 fell outside the other vertebrate group and formed an independent clade in the tree respectively, while zebrafish GAMT and frog GAMT were in the same clade (Fig. 1).

Three cDNA fragments for agat (355bp), gamt (417bp) and ct1 (625bp) were isolated by RT-PCR using the primers designed according to these sequences obtained from GenBank. After subcloning and sequencing, RNA probes were synthesized for whole mount in situ hybridization.

\section{Spatiotemporal expression of agat}

The temporal and spatial expression pattern of agat was determined from fertilization to $48 \mathrm{hpf}$ (Fig. 2). agat transcripts first appeared in the yolk syncytial layer (YSL) at gastrula stage (Fig. 2A,B). At early segmentation stage, strong agat expression persisted in the YSL and a weak expression also appeared in the mature somites (Fig. 2 C,D). As development proceeds, agat still expressed strongly in the YSL and weakly in the somites (Fig. 2 E,F). By 48 hpf, the expression of agat disappeared in the somites; however, strong expression was detected in the liver (Fig. $2 \mathrm{G}, \mathrm{H}$ ).

agatexpression pattern has been described in several other vertebrate species. It was expressed in the placenta and the yolk sac of mouse embryos. However, no expression was found in the embryonic tissues of mouse (Sandell et al., 2003). Similarly, human agat was also expressed in the placentas (Monk et al., 2006). Braissant et al. (2005) have reported agat expression in the hepatic primordium of rat embryos at E12.5. High levels of agat expression were also detected in kidney,
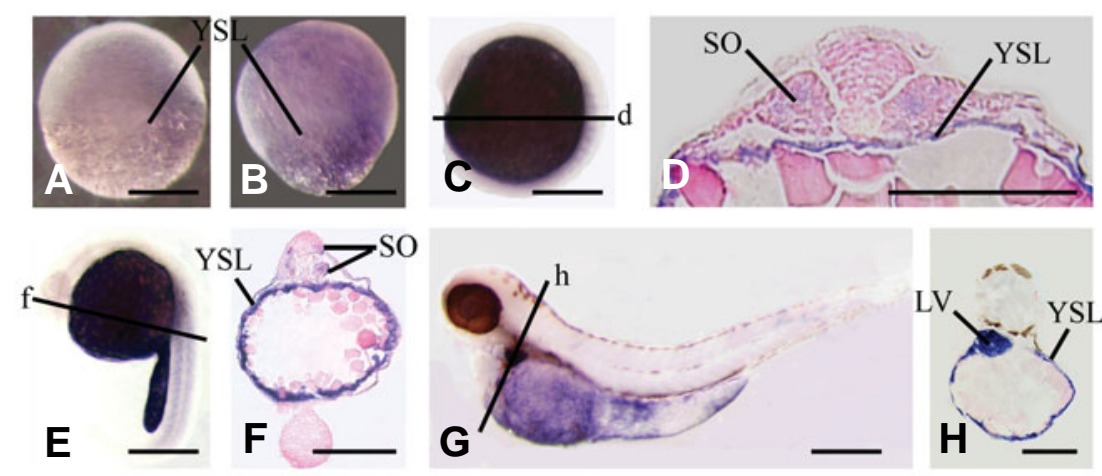

Fig. 2. Expression of agat in zebrafish embryos. Left side views for the whole mount, except where noted, with anterior up and dorsal to the right. (A) 50\%-epiboly stage, showing that agat mRNA was first detected in the YSL. (B) $70 \%$-epiboly stage, showing increased expression in the YSL. (C) 8-somite stage (13 h), showing agat expression in mature somites and the YSL. (D) Cross section of (C) through the line $d$. (E) 25-somite stage (21.5 h), showing strong expression in the YSL and weak expression in the mature somites. (F) Cross section of (E) through the line $f$. (G) Long-pec stage (48 h), left side view, with dorsal side up and anterior to the left. Expression of agat disappeared in the somites, but persisted in the YSL and appeared in the liver. (H) Cross section of (G) through the line $h$, showing strong expression in the liver and the YSL. LV, liver; SO, somite; YSL, yolk syncytial layer. Scale bar: $250 \mu$ m. 
Fig. 3. Expression of gamt in zebrafish embryos. Left side views for the whole mount, except where noted, with anterior up and dorsal to the right. (A) Early cleavage stage, side view. Weak expression was found in the center of the yolk. (B) Bud stage (10 h), ventral view. Expression of gamt appeared in the YSL. (C) 8-somite stage (13 h), showing gamt expression in the YSL. (D) Cross section of (C) through the line $d$. (E) 15-somite stage (16.5 h); gamt is expressed in the YSL and the mature somites. (F) Cross section of (E) through the line $f$. (G) Enlargement of the gray shadow area $g$ in (F), showing gamt expression in the YSL and the somites. (H) 25-somite stage $(21.5 \mathrm{~h})$, showing increased expression in the somites. (I) Long-pec stage (48 h). (J) Cross section of (I) through the line $j$. Staining of gamt disappeared in the somites, but persisted in the YSL and appeared in the liver and the gut. G, gut; LV, liver; SO, somite; YK, yolk; YSL, yolk syncytial layer. Scale bar: 80 $\mu \mathrm{m}$ for $(G)$ and $250 \mu \mathrm{m}$ for others.

Fig. 4. Expression of $\boldsymbol{c t} 1$ in zebrafish embryos. Left side views for the whole mount, except where noted, with anterior up and dorsal to the right. (A) 4-cell stage, animal pole view. (B) $70 \%$-epiboly stage. (C) $90 \%$ epiboly stage. (D) 8-somite stage (13 h). ct1 mRNA was distributed ubiquitously in the embryonic tissues in (AD) while no signal was found in the YSL. (E) 15-somite stage (16.5 h). (F) Ventral view of (E). (G) Dorsal view of (E). The expression was restricted in the somites, the optic vesicles and the CNS. (H) Prim-5 stage (24 h). (I) Dorsal view of $(H)$. The expression of ct1 mRNA persisted in both the newly formed and the matrue somites. (J) Cross section of $(H)$ through the line j, showing the expression in the somites. (K) Prim-15 stage (30 h), showing ct1 mRNA in the somites. (L) Long-pec stage $(48 \mathrm{~h})$, showing the expression of $\mathrm{ct} 1$ in the foregut. (M) Cross section of $(L)$ through the line $m$, showing ct1 expression in the epithelial cells of the foregut. CNS, central nervous system; G, gut; OV, optic vesicle; SO, somite. Scale bar: $80 \mu \mathrm{m}$ for (J) and (M), 250 $\mathrm{m}$ for others.
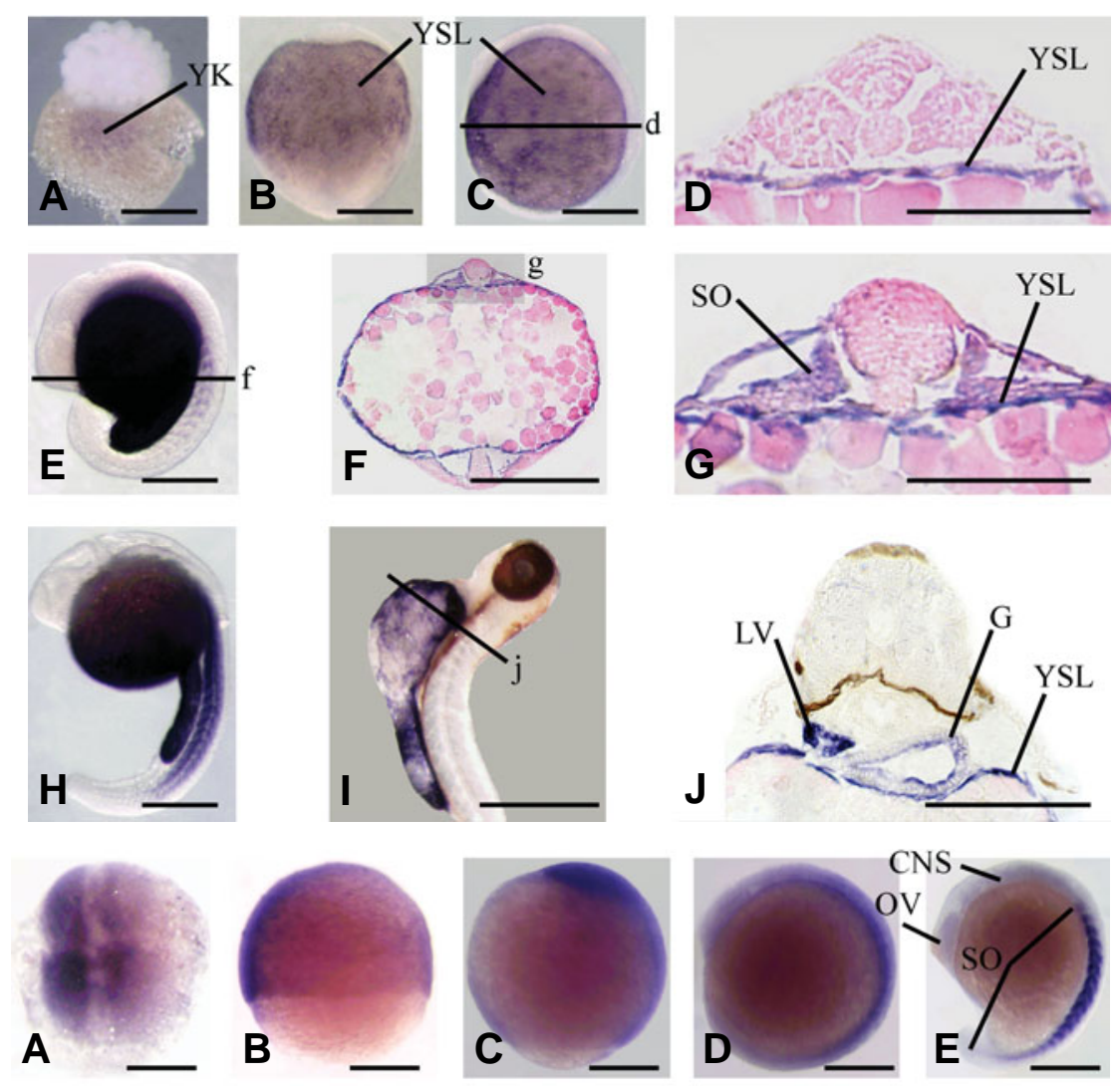

A
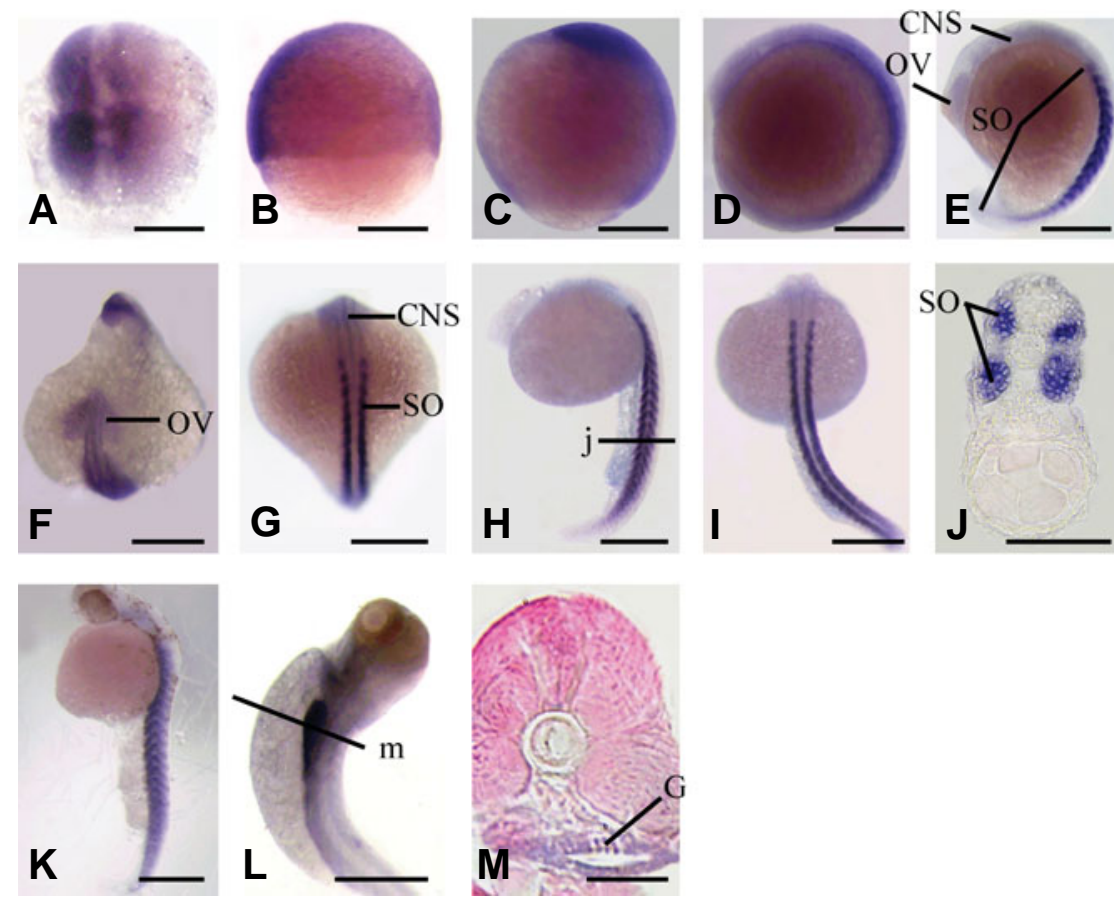

tissues (Fig. 3 B-D). At about 16 hpf, gamtexpression could be detected in the mature somites besides the YSL (Fig. $3 \mathrm{E}-\mathrm{G}$ ). The expression in the somites was gradually up regulated from $17 \mathrm{hpf}$ to $22 \mathrm{hpf}$ stage (Fig. 3H). Like agat, gamt expression in the somites disappeared at $48 \mathrm{hpf}$, but persisted in the YSL and appeared in the liver (Fig. $3 \mathrm{I}, \mathrm{J}$ ). However, gamtmRNA was also detected in the epithelial cells of the gut where no agatmRNA was found (Fig. 3J).

Similar pattern of expression could be noted between zebrafish gamt and rat gamt (Braissant et al., 2005). Both of them were detected in liver, muscles and gut. But the expression of rat gamt was also detected prominently in CNS and pancreas while zebrafish gamt was not.

\section{Spatiotemporal expression of ct1}

ct1 exhibited overlapping and distinct expression pattern compared with agat and gamt. ct 1 was initially expressed ubiquitously in the embryo from cleavage to early segmentation stage (Fig. 4 A-D). Then it was gradually restricted in the somites, the optic vesicles and the central nervous system (CNS) (Fig. 4 E-G). By prim-5 and prim-15 stage, ct1 expression was strongly expressed in both the newly formed and mature somites (Fig. $4 \mathrm{H}-\mathrm{K}$ ). By $48 \mathrm{hpf}$, the ct 1 transcripts decreased to a much weaker extent in the somites, but strongly 
appeared in the foregut (Fig. 4 L,M). ct1 expression has been reported in CNS, choroid plexus, dorsal aorta, hepatic primordium, skeletal muscles, epidermis and dermis, kidney, lung, stomach and intestine epithelial cells of rat embryos (Braissant et al., 2005). However, ct1 expression in zebrafish embryos was restricted to a fewer tissues, including somites, CNS, optic vesicle and gut.

Several interesting differences were noticed between the expression patterns of agat, gamt and ct1 in zebrafish embryos. First, unlike agatand/or gamt, no transcripts of $c t /$ were detected in the yolk, the YSL or the liver. Second, ct1 expression appeared in the CNS while agat and gamtdid not. Finally, high level of ct1 expression was detected in all the somites, while weak agat and gamt expressions were detected only in the mature somites. Thus, our results show that agat, gamt and ct1 genes exhibit unique expression pattern during zebrafish embryogenesis.

The expressions of agatand gamt in zebrafish are detected in YSL before $48 \mathrm{hpf}$ and in the liver after $48 \mathrm{hpf}$. This may suggest that zebrafish YSL and liver at different developmental stages are important tissues related to creatine synthesis. In addition, the somites during segmentation stage may be also involved in this process. In zebrafish, the expression of $c t 1$ is detected in CNS while agat and/or gamt are not. It implies that fish CNS may not function in creatine synthesis but just transport creatine to supply itself for energy.

Compared with their homologues during rat embryonic development (Braissant et al., 2005), there is a similar expression pattern of agat, gamtand $c t 1$ between zebrafish and rat embryos. They are all expressed in skeletal muscle. In addition, agat and gamt are expressed in liver. On the other side, there are differences of these gene expression patterns between two species. For example, three genes are expressed in the CNS of rat embryo while only $c t 1$ is expressed in the CNS of zebrafish embryo. agat gene is strongly expressed in rat kidney and pancreas, while no expression is found in the same organs of zebrafish embryo. Among these three genes related to creatine metabolism, the spatiotemporal expression of $c t 1$ shows a remarkable conservation between fish and mammal. It implies that the similar mechanism of creatine transport may occur in fish and mammal.

The expressions of muscle-specific creatine kinase and brain subtype creatine kinase have been reported during zebrafish development (Xu et al., 2000, Dickmeis et al., 2001). Our data show that three primary elements related to creatine metabolism exist in zebrafish. Together with previous report (Xu et al., 2000, Dickmeis et al., 2001), we suggest that a creatine synthesis and transport system as in mammal may similarly exist in fish. Since zebrafish is easy to maintain, manipulate and observe in the lab, it may provide a model system for human disease study (Dodd et al., 2000, Dooley and Zon, 2000). Further functional analysis of these molecules in zebrafish should be useful for understanding the mechanism of human creatine deficiency syndromes.

\section{Experimental Procedures}

Cloning, sequence alignments and phylogenetic analyses

The RT-PCR reactions were carried out on RNA extracted from $24 \mathrm{hpf}$ zebrafish embryos according to manufacturer's instructions "Promega". The primers were as follows:

agat 5'-TACGGCGGTCAGACATTC-3' and

5'-GGTAATCCTGGTCGTAGAGC-3';

\section{gamt 5'-CGTTCACGCACTCCGCATTTG-3' and \\ 5'-AGGGTTGGGGCGACCTCTTCC-3'; \\ ct1 5'-AACTCCACATTCGGCAACCT-3' and \\ 5'-GAGCCACGGGCATCATAGA-3'.}

The PCR product was 355 bp corresponding to position 379-733 of the cDNA (AAH56747) for agat, 417 bp and located in the region corresponding to 5-421 of the cDNA (NM_205741) for gamt, $625 \mathrm{bp}$ corresponding to position 625-1249 of the cDNA (XM_690840) for ct1. PCR products were subcloned into pGEM-T Easy vectors (Promega, Madison, WI) and sequenced. Alignments of sequences and phylogenetic analyses were performed with ClustalX 1.81 and Treepuzzle 5.0 software.

\section{Probes synthesis and whole mount in situ hybridization}

Sense and anti-sense RNA probes were synthesized using the digoxigenin-UTP (DIG) in vitro transcription kit with SP6 and T7 polymerases (Roche Applied Science, Indianapolis, IN) on the abovementioned cDNA clones. In situ hybridizations were performed as described (see http://zfin.org/zf_info/zfbook/chapt9/9.82.html), followed by sectioning.

\section{Acknowledgements}

We express appreciation to Dr. Shaojun Du, Center of Marine Biotechnology, University of Maryland Biotechnology Institute, for reading and revising the manuscript. This project was funded by grants from CNSF to Hongwei Zhang (30270693 30570967 ).

\section{References}

ALMEIDA, L.S., VERHOEVEN, N.M., ROOS, B., VALONGO, C., CARDOSO, M.L., VILARINHO, L., SALOMONS, G.S. and JAKOBS, C. (2004). Creatine and guanidinoacetate: Diagnostic markers for inborn errors in creatine biosynthesis and transport. Mol Genet Metab 82: 214-219.

BATTINI, R., ALESSANDRI, M.G., LEUZZI, V., MORO, F., TOSETTI, M., BIANCHI, M.C. and CIONI, G. (2006). Arginine:Glycine amidinotransferase (agat) deficiency in a newborn: Early treatment can prevent phenotypic expression of the disease. J Pediatr 148: 828-830.

BRAISSANT, O., HENRY, H., VILLARD, A.M., SPEER, O., WALLIMANN, T. and BACHMANN, C. (2005). Creatine synthesis and transport during rat embryogenesis: Spatiotemporal expression of agat, gamt and ct1. BMC Dev Bio/5: 9.

CECIL, K.M., SALOMONS, G.S., BALL, W.S., JR., WONG, B., CHUCK, G., VERHOEVEN, N.M., JAKOBS, C. and DEGRAUW, T.J. (2001). Irreversible brain creatine deficiency with elevated serum and urine creatine: A creatine transporter defect? Ann Neuro/49: 401-404.

DICKMEIS, T., RASTEGAR, S., AANSTAD, P., CLARK, M., FISCHER, N., PLESSY, C., ROSA, F., KORZH, V. and STRAHLE, U. (2001). Expression of brain subtype creatine kinase in the zebrafish embryo. Mech Dev 109: 409412.

DODD, A., CURTIS, P.M., WILLIAMS, L.C. and LOVE, D.R. (2000). Zebrafish: Bridging the gap between development and disease. Hum Mol Genet9:24432449.

DOOLEY, K. and ZON, L.I. (2000). Zebrafish: A model system for the study of human disease. Curr Opin Genet Dev 10: 252-256.

LEUZZI, V., CARDUCCI, C., CARDUCCI, C., MATRICARDI, M., BIANCHI, M.C., DI SABATO, M.L., ARTIOLA, C. and ANTONOZZI, I. (2006). A mutation on exon 6 of guanidinoacetate methyltransferase (gamt) gene supports a different function for isoform a and b of gamt enzyme. Mol Genet Metab 87: 88-90.

MANCINI, G.M., CATSMAN-BERREVOETS, C.E., DE COO, I.F., AARSEN, F.K., KAMPHOVEN, J.H., HUIJMANS, J.G., DURAN, M., VAN DER KNAAP, M.S., JAKOBS, C. and SALOMONS, G.S. (2005). Two novel mutations in slc6a8 cause creatine transporter defect and distinctive $\mathrm{x}$-linked mental retardation in two unrelated dutch families. Am J Med Genet A 132: 288-295.

MONK, D., ARNAUD, P., APOSTOLIDOU, S., HILLS, F.A., KELSEY, G., STANIER, P., FEIL, R. and MOORE, G.E. (2006). Limited evolutionary conservation of imprinting in the human placenta. Proc Nat/ Acad Sci U S A 103: 6623-6628. 
SANDELL, L.L., GUAN, X.J., INGRAM, R. and TILGHMAN, S.M. (2003). Gatm, a creatine synthesis enzyme, is imprinted in mouse placenta. Proc Nat/ Acad SciUS A 100: 4622-4627.

SCHULZE, A. (2003). Creatine deficiency syndromes. Mol Cell Biochem 244: 143-150.

SCHULZE, A., HOFFMANN, G.F., BACHERT, P., KIRSCH, S., SALOMONS, G.S., VERHOEVEN, N.M. and MAYATEPEK, E. (2006). Presymptomatic treatment of neonatal guanidinoacetate methyltransferase deficiency. Neurology 67: 719-721.

STROMBERGER, C., BODAMER, O.A. and STOCKLER-IPSIROGLU, S. (2003). Clinical characteristics and diagnostic clues in inborn errors of creatine metabolism. J Inherit Metab Dis 26: 299-308.

SYKUT-CEGIELSKA, J., GRADOWSKA, W., MERCIMEK-MAHMUTOGLU, S. and STOCKLER-IPSIROGLU, S. (2004). Biochemical and clinical characteristics of creatine deficiency syndromes. Acta Biochim Po/51: 875-882.

VAN DER KNAAP, M.S., VERHOEVEN, N.M., MAASWINKEL-MOOIJ, P., POUWELS, P.J., ONKENHOUT, W., PEETERS, E.A., STOCKLERIPSIROGLU, S. and JAKOBS, C. (2000). Mental retardation and behavioral problems as presenting signs of a creatine synthesis defect. Ann Neuro/47:
540-543.

VERHOEVEN, N.M., GUERAND, W.S., STRUYS, E.A., BOUMAN, A.A., VAN DER KNAAP, M.S. and JAKOBS, C. (2000). Plasma creatinine assessment in creatine deficiency: A diagnostic pitfall. J Inherit Metab Dis 23: 835-840.

WYSS, M. and KADDURAH-DAOUK, R. (2000). Creatine and creatinine metabolism. Physiol Rev 80: 1107-1213.

XU, Y., HE, J., WANG, X., LIM, T.M. and GONG, Z. (2000). Asynchronous activation of 10 muscle-specific protein (msp) genes during zebrafish somitogenesis. Dev Dyn 219: 201-215.

ZHAO, H., CAO, Y. and GRUNZ, H. (2001). Expression of xenopus Iarginine:Glycine amidinotransferase (xat) during early embryonic development. Dev Genes Evo/211: 358-360.

Received: 5th September 2006 Reviewed by Referees: 26th October 2006 Modified by Authors and Accepted for Publication: 14th November 2006 Published Online: 9th February 2007

\section{Related Int. J. Dev. Biol. articles}

Cadherin-6 is required for zebrafish nephrogenesis during early development. Fumitaka Kubota, Tohru Murakami, Kenji Mogi and Hiroshi Yorifuji. Int. J. Dev. Biol. (2007) 51: 123-129.

Formation of a successional dental lamina in the zebrafish (Danio rerio): support for a local control of replacement tooth initiation. Ann Huysseune. Int. J. Dev. Biol. (2006) 50: 637-643.

Transient expression of apoaequorin in zebrafish embryos: extending the ability to image calcium transients during later stages of development. Chris Y. Cheung, Sarah E. Webb, Anming Meng and Andrew L. Miller. Int. J. Dev. Biol. (2006) 50: 561-569.

Expression of FoxP2 during zebrafish development and in the adult brain. Rina Shah, Olga Medina-Martinez, Li-Fang Chu, Rodney C. Samaco and Milan Jamrich. Int. J. Dev. Biol. (2006) 50: 435-438.

Cloning, expression and functional study of translation elongation factor 2 (EF-2) in zebrafish. Shu-hong Zhang, Ji-hua Yao, Huai-dong Song, Lu Wang and Jing-lun Xue. Int. J. Dev. Biol. (2006) 50: 399-403.

Developmental expression of the High Mobility Group B gene in the amphioxus, Branchiostoma belcheri tsingtauense. Xiangwei Huang, Lifeng Wang and Hongwei Zhang. Int. J. Dev. Biol. (2005) 49: 49-46.

Insulin-like growth factor (IGF) signalling is required for early dorso-anterior development of the zebrafish embryo. Edward Eivers, Karena Mccarthy, Catherine Glynn, Catherine M. Nolan and Lucy Byrnes. Int. J. Dev. Biol. (2004) 48: 1131-1140.

A moving wave patterns the cone photoreceptor mosaic array in the zebrafish retina. Pamela A. Raymond and Linda K. Barthel. Int. J. Dev. Biol. (2004) 48: 935-945.

Genetics of photoreceptor development and function in zebrafish. Motokazu Tsujikawa and Jarema Malicki. Int. J. Dev. Biol. (2004) 48: 925-934.

Scale development in fish: a review, with description of sonic hedgehog (shh) expression in the zebrafish (Danio rerio). Jean-Yves Sire and Marie-Andrée Akimenko. Int. J. Dev. Biol. (2004) 48: 233-247.

Skin development in bony fish with particular emphasis on collagen deposition in the dermis of the zebrafish (Danio rerio). Dominique Le Guellec, Ghislaine Morvan-Dubois and Jean-Yves Sire. Int. J. Dev. Biol. (2004) 48: 217-231. 
APPENDIX I

\section{Amino acid sequence alignment of AGAT (A), GAMT (B) and CT1 (C) in zebrafish and other species using} ClustalX software

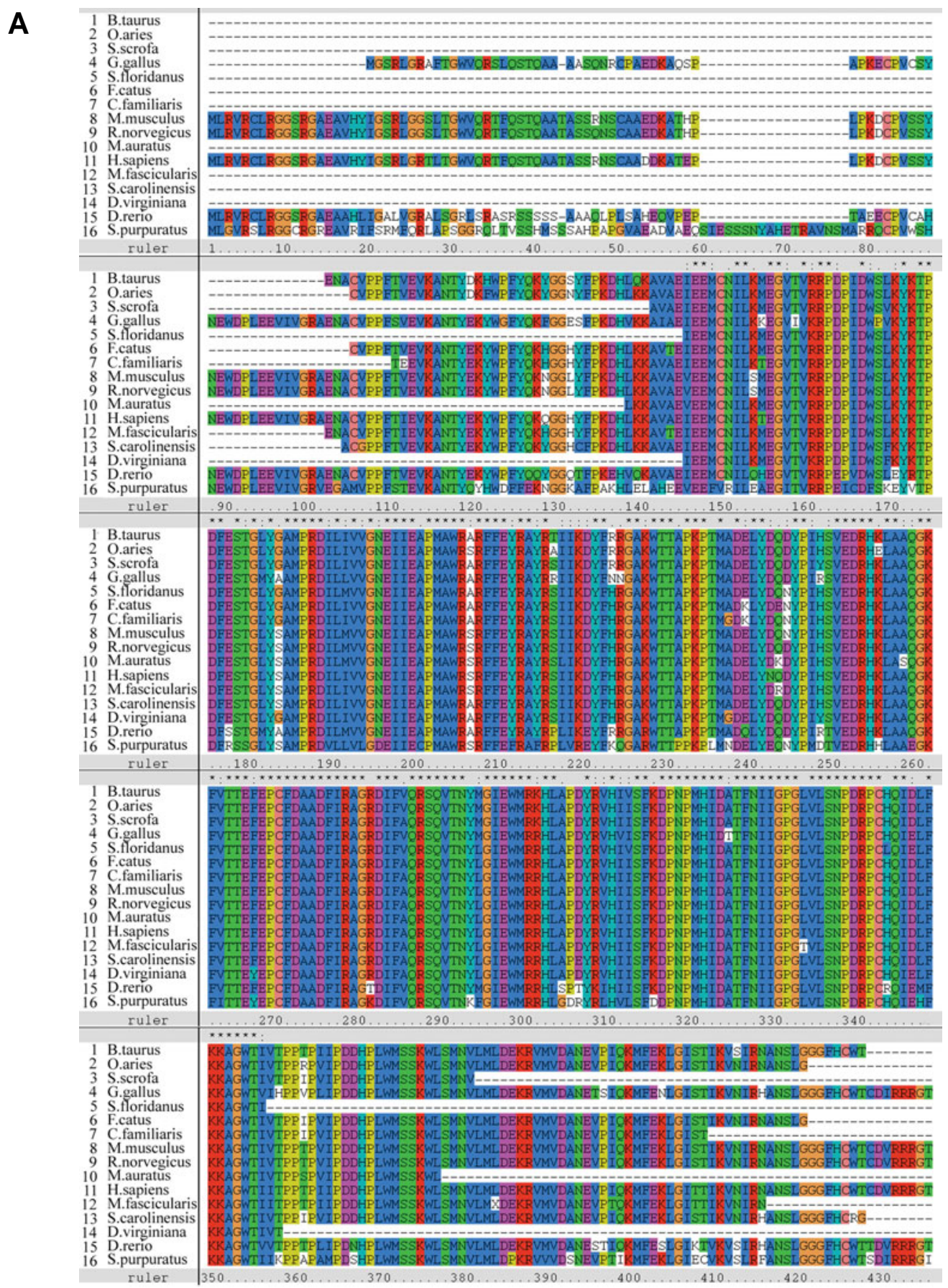

Color background indicates the amino acids that match the consensus. The asterisks indicate identical amino acids among all species. The following are the accession numbers of amino acid sequences. For AGAT: AAT39889 (Bos Taurus), AAT39898(Ovis aries), AAT39894 (Sus scrofa), NP_990076 (Gallus gallus), AAT39896 (Sylvilagus floridanus), AAT39888 (Felis catus), AAT39890 (Canis familiaris), AAH03879 (Mus musculus), NP_112293 (Rattus norvegicus), AAT39891 (Mesocricetus auratus), AAB29892 (Homo sapiens), AAT39892 (Macaca fascicularis), AAT39899(Sciurus carolinensis), AAT39895 (Didelphis virginiana), AAH56747 (Danio rerio), XP_786560 (Strongylocentrotus purpuratus). For GAMT: AAH45001 (Xenopus laevis), NP991304 (Danio rerio), ABA00513 (Branchiostoma belcheri), NP-0000147 (Homo sapiens isoform a), NP-620279(Homo sapiens isoform b), NP-034385 (Mus musculus), NP_036925 (Rattus norvegicus). For CT1: P28570 (Rattus norvegicus), Q8VBW1(Mus musculus), P48029 (Homo sapiens), P31661(Oryctolagus cuniculus), 018875 (Bos taurus), XP_549362 (Canis familiaris), XP_695932 (Danio rerio). 
B

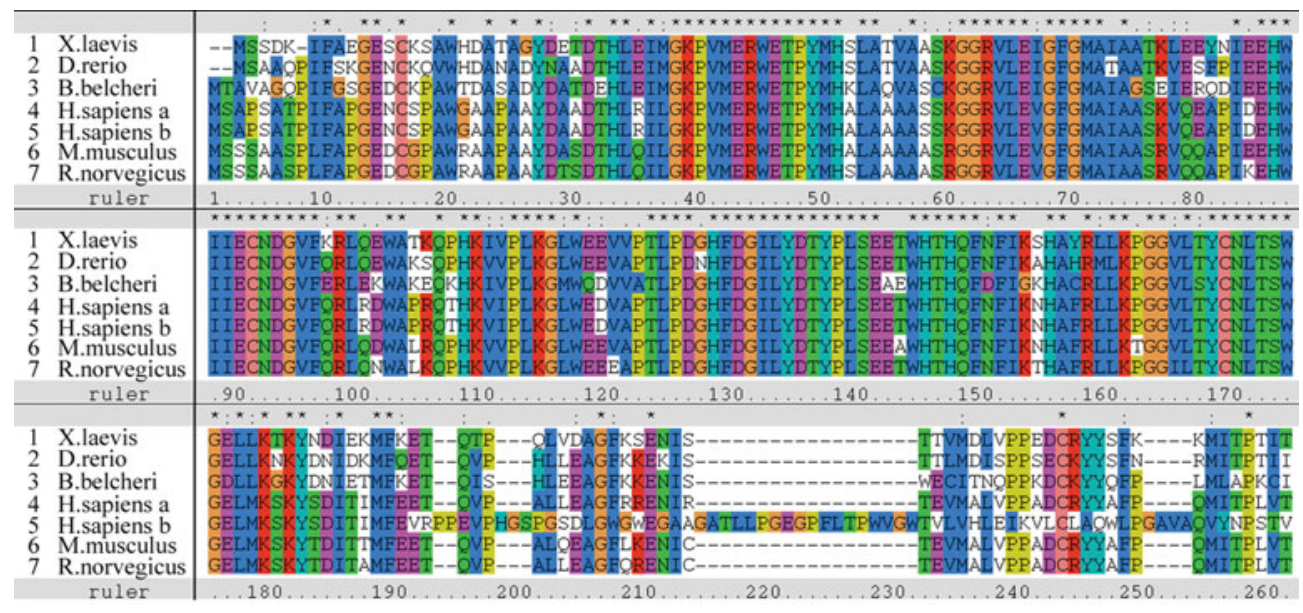

C

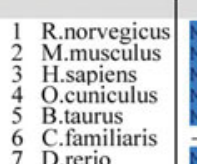

7 D.rerio

ruler

$\begin{array}{ll}1 & \text { R.norvegicus } \\ 2 & \text { M.musculus } \\ 3 & \text { H.sapiens }\end{array}$

3 H.sapiens

5 B.taurus

6 C.familiaris

7 D.rerio ruler

1 R.norvegicus

M.musculus

H.sapiens

O.cuniculus

6 C.familiaris

6 C.famili

ruler

1 R.norvegicus

M.musculus

3 H.sapiens

4 O.cuniculus

5 B.taurus

6 C.familiar

7 D.rerio ruler

1 R.norvegicus

H.sapiens

4 O.cuniculus

5 B.taurus

6 C.familia

7 D.rerio

ruler

1 R.norvegicus

M.musculus

3 H.sapiens

4 O.cuniculus

5 B.taurus

6 C.familiaris

7 D.rerio ruler

1 R.norvegicus

2 M.musculus

H.sapiens

O.cunicul

6 C.familiaris

7 D.rerio ruler

R.norvegicus

M.musculus

H.sapiens

O.cunicul

B.taurus

6 C.familia

7 D.rerio
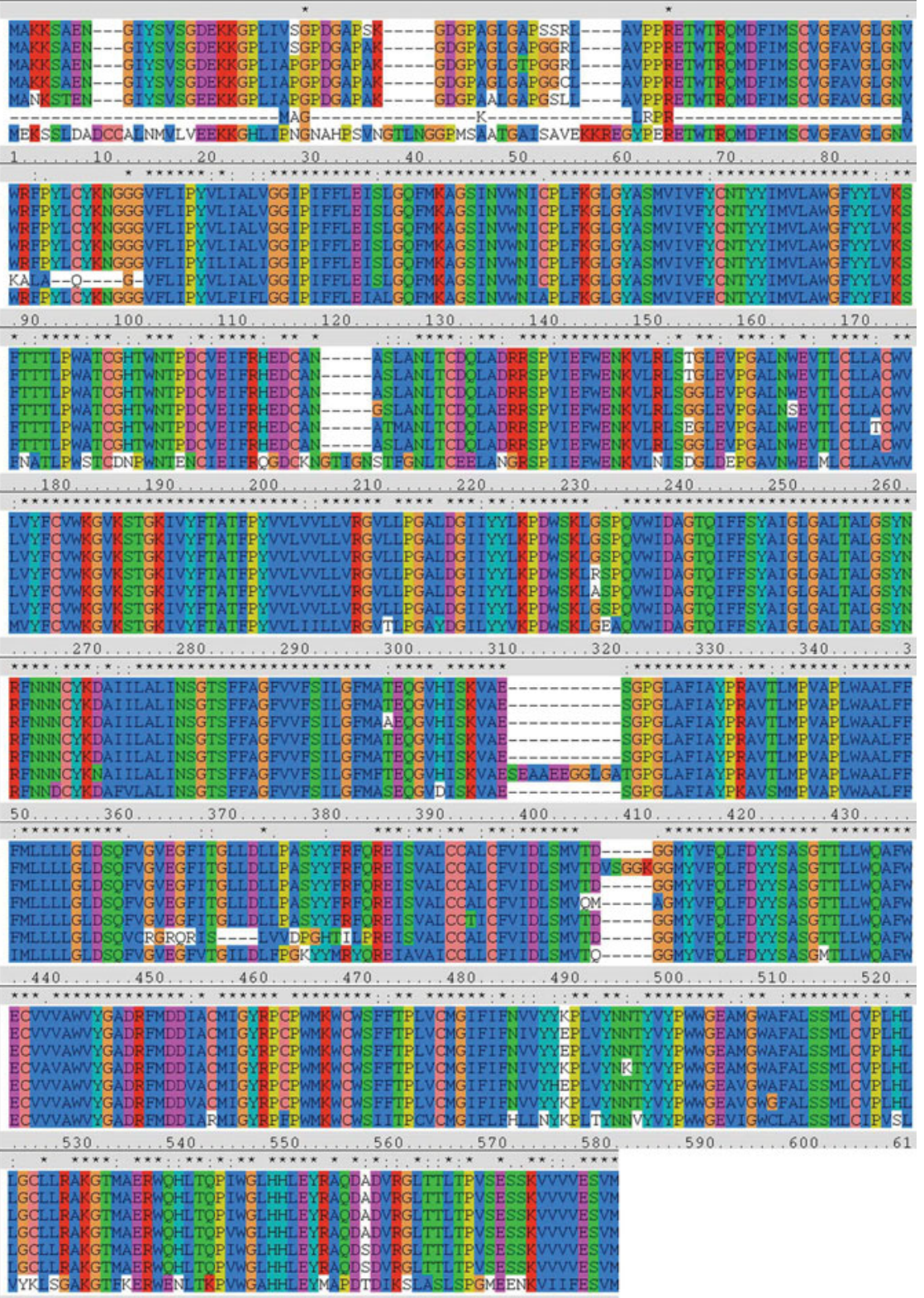